\title{
Erratum
}

\section{Integrated specific star formation rates of galaxies, groups, and clusters: a continuous upper limit with stellar mass?}

\author{
G. Feulner ${ }^{1,2,3}$, U. Hopp ${ }^{1,2}$, and C. S. Botzler ${ }^{1,4,5}$ \\ 1 Universitäts-Sternwarte München, Scheinerstraße 1, 81679 München, Germany \\ e-mail: feulner@usm.lmu.de \\ 2 Max-Planck-Institut für extraterrestrische Physik, Giessenbachstraße 1, 85748 Garching, Germany \\ 3 Potsdam-Institut für Klimafolgenforschung, Postfach 6012 03, 14412 Potsdam, Germany \\ 4 University of Auckland, Private Bag 92019, Morrin Road, Glen Innes, Auckland, New Zealand \\ 5 University of Canterbury, Private Bag 4800, Christchurch, New Zealand
}

A\&A, 451, L13-L16 (2006), DOI: 10.1051/0004-6361:20065082

Key words. galaxies: evolution - galaxies: formation - galaxies: fundamental parameters - galaxies: high-redshift galaxies: clusters: general - errata, addenda

Unfortunately, in Eq. (5) of our aforementioned paper, which describes the functional form of the upper envelope in the specific star formation rate $\mathcal{S}$ as a function of stellar mass $\mathcal{M}$, there are two typesetting errors. First, the parameter stellar masses $\mathcal{M}$ should be replaced with the expression $\log \mathcal{M}$, and secondly, there is a sign error in the exponential function. The correct equation is

$\mathcal{S}=\mathcal{S}_{0} 10^{(1+\alpha) \log \mathcal{M}_{*}} \exp \left(-10^{\log \mathcal{M}_{*}-\log \mathcal{M}_{0}}\right)$

where $\mathcal{S}_{0}$ denotes the normalisation, $\mathcal{M}_{0}$ is the location of the break, and $\alpha$ is the slope at lower stellar masses. These errors do not affect any of the conclusions of our original paper, since the correct form of the equation was in fact used in all computations. 